\title{
Downregulated SIRT6 and upregulated NMNAT2 are associated with the presence, depth and stage of colorectal cancer
}

\author{
JIA QI $^{1}$, CHUNHUI CUI ${ }^{1}$, QUANWEN DENG ${ }^{2}$, LIFENG WANG $^{3}$, RIHONG CHEN ${ }^{1}$, \\ DUANYANG ZHAI ${ }^{1}$, LANG XIE ${ }^{1}$ and JINLONG YU ${ }^{1}$ \\ ${ }^{1}$ Department of General Surgery, Zhujiang Hospital of Southern Medical University, Guangzhou, Guangdong 510280; \\ ${ }^{2}$ Department of Pharmacy, Nanfang Hospital of Southern Medical University, Guangzhou, Guangdong 510515; \\ ${ }^{3}$ Department of Gastroenterology, Zhujiang Hospital of Southern Medical University, \\ Guangzhou, Guangdong 510280, P.R. China
}

Received July 22, 2016; Accepted January 8, 2018

DOI: $10.3892 / \mathrm{ol} .2018 .9400$

\begin{abstract}
Colorectal cancer (CRC) is an important cause of morbidity and mortality worldwide, and is difficult to detect in its early stages. Diagnostic and prognostic biomarkers are required, which may also be the basis for improving the targeted therapy for CRC. Sirtuin 6 (SIRT6) is a member of the sirtuin family of gene regulators, which have specific functions in genomic stability, gene transcription and energy metabolism in tumorigenesis. Nicotinamide mononucleotide adenylyltransferase 2 (NMNAT2) is a metabolic enzyme which can be deacetylated by sirtuins. In this study, tissue samples from 29 patients with histologically confirmed CRC of varying grade and stage were studied for SIRT6 and NMNAT2 expression by western blotting and reverse transcription-quantitative polymerase chain reaction. Immunohistochemistry was performed for SIRT6 and NMNAT2 expression in 113 paired (CRC and adjacent) tissue sections. SIRT6 protein and mRNA expression levels were significantly reduced in CRC tissues; NMNAT2 protein and mRNA expression levels were significantly increased in $\mathrm{CRC}$ tissues $(\mathrm{P}<0.01)$. A negative correlation between the expression of SIRT6 and NMNAT2 in CRC tissue samples was identified $(\mathrm{r}=-0.246, \mathrm{P}<0.01)$. The reduced expression of SIRT6 and increased expression of NMNAT2 were associated with the tumor depth invasion, stage, differentiation grade (SIRT6 only) and the presence of lymph node metastasis $(\mathrm{P}<0.05)$. In conclusion, the findings of the present
\end{abstract}

Correspondence to: Dr Jinlong Yu, Department of General Surgery, Zhujiang Hospital of Southern Medical University, 253 Industrial Avenue, Guangzhou, Guangdong 510280, P.R. China

E-mail: projinlongyu@sina.com

Abbreviations: CRC, colorectal cancer; NAD, nicotinamide adenine dinucleotide; NMNAT2, nicotinamide mononucleotide adenylyltransferase 2; SIRT6, sirtuin 6

Key words: colorectal carcinoma, nicotinamide mononucleotide adenylyltransferase 2 , sirtuin 6 preliminary study demonstrated that the increased expression of NMNAT2 and reduced expression of SIRT6 may be associated with the progression of CRC. The downregulation of SIRT6 may promote the expression of NMNAT2. Further studies are indicated on the role of NMNAT2 and SIRT6 as potential diagnostic and prognostic biomarkers and as targets for therapy in CRC and other malignant tumors.

\section{Introduction}

Colorectal cancer (CRC) is one of the most common types of solid tumor, with $\sim 1.2$ million new confirmed diagnoses of CRC annually worldwide and with $55 \%$ of cases occurring in economically developed countries (1). Changes in lifestyle, population growth and the aging population have made CRC an increasingly global health problem (2). Patients with CRC have a poor prognosis; $15 \%$ of patients present with metastases at the time of initial diagnosis, and $\sim 35 \%$ of patients diagnosed with CRC ultimately develop metastasis $(3,4)$. Despite the recent use of neoadjuvant chemotherapy (NCT) or NCT in combination with targeted agents, which has resulted in an improvement in the overall survival rate, $\mathrm{CRC}$ remains one of the leading causes of cancer mortality, with $>600,000$ mortalities occurring due to CRC annually worldwide $(5,6)$. Identifying specific molecules associated with CRC may facilitate the development of diagnostic and prognostic biomarkers, and more specific targets for therapy, in order to further improve the clinical outcome of patients with CRC.

The sirtuins (SIRTs) are a conserved family of nicotinamide adenine dinucleotide (NAD)-dependent histone deacetylases composed of seven SIRT isoforms (SIRT1-7) in mammalian genomes $(7,8)$ The nuclear protein SIRT6 is a member of the SIRT family, which has been demonstrated to exert diverse cancer-associated functions, including in genomic stability, DNA repair, gene transcription repression and stress resistance, by multiple molecular mechanisms (9). SIRT6 is a chromatin-bound factor that promotes genomic integrity by de-acetylating histone H3 lysine 9 (H3K9) and 56; SIRT6-mediated histone de-acetylation contributes to the repair of DNA double-strand breaks (10-12). SIRT6 may act as a chromatin-bound factor to protect cells from genomic 
instability, including in non-transformed cells $(13,14)$. SIRT6 has also been reported to function at the gene expression level via the recruitment of transcription factors, including $\mathrm{NF}-\kappa \mathrm{B}$ and c-Jun, and may deacetylate H3K9 at the site of gene promoters $(15,16)$ A previous study identified SIRT6 as a novel molecular mechanism for controlling energy metabolism in cancer cells (17). In 2012, the Cancer Cell Line Encyclopedia reported that the SIRT6 gene is deleted in $35 \%$ of the 1,000 human cancer cell lines recorded in their database, including in $29 \%$ of CRC cell lines (18). However, the role of SIRT6 in CRC and the molecular mechanisms by which it acts as a regulator of cancer-associated metabolism remain unknown.

Nicotinamide mononucleotide adenylyltransferase 2 (NMNAT2) is an isoform of NMNAT, which catalyzes a vital step in the synthesis of NAD (19). NMNAT2 is a sensitive enzyme marker for NAD levels that reflects the intracellular redox equilibrium and cellular energy state, acting as a sensor for cells with a high energy demand, including cancer cells $(20,21)$. Although the associated biological mechanism remains largely unknown, there is increasing evidence that NMNAT2 promotes the survival of cancer cells by accelerating glycolysis (22). SIRT6 controls the expression of multiple glycolytic genes, including HIF-1 $\alpha$ (23).

Given the evidence of the possible roles for SIRT6 and NMNAT2 in human cancer, including of SIRT6 in the control of energy metabolism by acting either as a substrate for, or regulator of, NMNAT2 (24), the present study was performed to determine the expression of SIRT6 and NMNAT2 in different grades and stages of CRC.

\section{Materials and methods}

Ethical statement. All patients included in this study were informed of the study protocol and requirements, and provided signed informed consent to participate in the study. The study, including the protocols for collection of tissue specimens and analysis of clinical information, was approved by the Institutional Review Board of Nanfang Hospital and Zhujiang Hospital of the Southern Medical University (Guangzhou, China).

Inclusion and exclusion criteria. All patients included in the present study met the following criteria: i) A histologically confirmed diagnosis of primary CRC; ii) treatment with radical surgical resection; iii) adequate tissue specimens available for tumor and normal tissue. Patients were excluded from the study if they met the following exclusion criteria: i) A history of other types of malignant diseases or metastatic $\mathrm{CRC}$; ii) receipt of preoperative radiotherapy, chemotherapy and other treatments.

Tissue specimens and clinical data collection. A total of 113 patients with CRC were enrolled in the study. The patients underwent curative surgical resection at the Nanfang Hospital and Zhujiang Hospital of the Southern Medical University between January 2010 and May 2016. From these patients, it was possible to obtain tissue from 29 fresh samples between January 2016 and May 2016, from which tissue sections were made and the extraction of mRNA and proteins was performed.
The 113 patients included 65 males and 48 females aged between 30 and 91 years, with a median age of 64 years. Patients were classified into four stages (stage I, 36; stage II, 30; stage III, 29; and stage IV, 18) according to the Union for International Cancer Control Tumor-Node-Metastasis (TNM) staging system (25). Tumors were graded according to the World Health Organization system (26) as well differentiated $(n=18)$, moderately differentiated $(n=87)$ or poorly differentiated $(n=8)$. The following clinical data were also recorded and analyzed in the study: Tumor size, depth of invasion, lymph node metastasis and distant metastasis. The histological diagnosis, classification and tissue sampling was performed by two experienced senior pathologists.

From the surgically resected colectomy specimens, pathologists sampled tumor tissue as well as adjacent normal colorectal tissue that was confirmed to be free from infiltration by $\mathrm{CRC}$ and located $>5 \mathrm{~cm}$ beyond the tumor margin. Fresh tissue samples that were sampled following resection were frozen in liquid nitrogen and stored at $-80^{\circ} \mathrm{C}$ until analysis was performed.

Reverse transcription-quantitative polymerase chain reaction (RT-qPCR) analysis of gene transcription. A total of 29 samples of tissue RNA was extracted with TRIzol reagent (Invitrogen; Thermo Fisher Scientific, Inc., Waltham, MA, USA) and RNA concentration was measured by using a NanoDrop 1000 spectrophotometer (Thermo Fisher Scientific, Inc., Penfield, NY, USA). PrimeScript RT master mix (Takara Bio, Inc., Otsu, Japan) was added to synthesize the first strand of cDNA using the Reverse Transcription system (Promega Corporation, Madison, WI, USA) according to the manufacturer's protocol.

RT-qPCR was performed using the 7500 FastReal-Time Two-step PCR system (Applied Biosystems; Thermo Fisher Scientific, Inc.) with the SYBR-Green qPCR master mix (Takara, Tokyo, Japan). The amplification protocol commenced with a 30 -sec pre-denaturation step at $95^{\circ} \mathrm{C}$, followed by 40 cycles of denaturation at $95^{\circ} \mathrm{C}$ for $5 \mathrm{sec}$, and annealing and extension at $60^{\circ} \mathrm{C}$ for $34 \mathrm{sec}$. Subsequent verification of product specificity was conducted in the melting stage, ending with inactivation at $95^{\circ} \mathrm{C}$ for $15 \mathrm{sec}$.

Quantitative gene expression data were normalized to the expression level of GAPDH and the analysis of each sample was performed in triplicate. The primers for human genes were designed by PrimerBank public database and purchased from Generay Biotech Co., Ltd. (Shanghai, China). The human-specific primers for each gene are listed in Table I. Relative quantification was performed using the $2^{-\Delta \Delta C q}$ method (27).

Western blotting analysis of gene translation. A total of 29 sample pairs of CRC and adjacent non-cancer colorectal tissues were lysed in radioimmunoprecipitation buffer (Nanjing KeyGen Biotech Co., Ltd., Nanjing, China) containing a protease inhibitor cocktail, phenylmethanesulfonyl fluoride and DL-dithiothreitol (Sigma-Aldrich; Merck KGaA, Darmstadt, Germany) in order to extract the protein from the samples. Following quantification with a bicinchoninic acid protein assay kit (Thermo Fisher Scientific, Inc.), the proteins were mixed with $5 \mathrm{X}$ SDS-PAGE loading buffer (Invitrogen; 
Table I. Gene primers used for the polymerase chain reaction analysis of SIRT6 and NMNAT2 in colorectal carcinoma tumors.

\begin{tabular}{lll}
\hline Gene & Forward primer, 5'-3' & \multicolumn{1}{c}{ Reverse primer, 5'-3' } \\
\hline SIRT6 & CCCACGGAGTCTGGACCAT & CTCTGCCAGTTTGTCCCTG \\
NMNAT2 & TGTCCACGACTCCTATGGAAA & GTCCGATCACAGGTGTCATGG \\
GAPDH & GGAGCGAGATCCCTCCAAAAT & GGCTGTTGTCATACTTCTCATGG
\end{tabular}

SIRT6, sirtuin 6; NMNAT2, nicotinamide mononucleotide adenylyltransferase 2.

Thermo Fisher Scientific, Inc.) in a water bath heated to $95^{\circ} \mathrm{C}$ for $5 \mathrm{~min}$ to denature the proteins. A total of $30 \mu \mathrm{g}$ protein was loaded per lane and separated by $12 \%$ SDS-PAGE. Following SDS-PAGE separation, proteins were transferred to polyvinyldifluoridine membranes.

Subsequent to blocking with $10 \%$ bovine serum albumin (Gibco; Thermo Fisher Scientific, Inc.) at room temperature for $1 \mathrm{~h}$, the membranes were incubated overnight at $4^{\circ} \mathrm{C}$ with primary antibodies, including anti-SIRT6 (cat no. ab62739; dilution, 1:800; Cell Signaling Technology, Inc., Danvers, MA, USA), anti-NMNAT2 (cat no. sc-515206; dilution, 1:1,000; Santa Cruz Biotechnology, Inc., Dallas, TX, USA) and anti-GAPDH (cat no. ab125247; dilution, 1:2,000, Abcam, Cambridge, MA, USA), followed by washing with TBS-T buffer [including $10 \mathrm{mM}$ Tris- $\mathrm{HCl}(\mathrm{Ph} 7.4), 150 \mathrm{mM} \mathrm{NaCl}$ and $0.05 \%$ Tween-20] and incubation with secondary antibodies [goat anti-mouse IgG (cat no. ab6789; dilution, 1:5,000) and goat anti-rabbit IgG (cat no. ab6721; dilution, 1:10,000), obtained from Abcam] at room temperature for $1 \mathrm{~h}$. GAPDH served as an endogenous protein control. The immunoreactive bands were visualized with enhanced chemiluminescence (Beyotime Institute of Biotechnology, Shanghai, China) and the gray values of each band were detected using Quantity One software version 4.6.2 (Bio-Rad Laboratories, Inc., Hercules, CA, USA).

Immunohistochemical detection of protein expression in tissue sections. FFPE tumor and adjacent tissues were sectioned onto glass slides as $4-\mu \mathrm{m}$ tissue sections. These sections were de-waxed by dipping in xylene twice (10 min each) at room temperature and rehydrated through a graded alcohol series subsequent to heating overnight at $60^{\circ} \mathrm{C}$. The endogenous tissue peroxidase activity was blocked with $3 \%$ hydrogen peroxide, and pressure- and heat-induced antigen retrieval was performed in citrate buffer $(\mathrm{pH}$ 6.0; Wuhan Boster Biological Technology Co., Ltd., Wuhan, China) for $3 \mathrm{~min}$. The tissue sections were exposed to $10 \%$ goat serum albumin (OriGene Technologies, Inc., Rockville, MD, USA) blocking solution (for $30 \mathrm{~min}$ at room temperature) prior to incubation in the primary antibody solution of anti-SIRT6 (dilution, 1:150; Abcam), or anti-NMNAT2 (dilution, 1:100; Santa Cruz Biotechnology, Inc.) overnight at $4^{\circ} \mathrm{C}$, then the slides were incubated with Biotin-conjugated goat anti-mouse/rabbit IgG secondary antibody (cat no. SP-9000; ready-to-use; OriGene Technologies, Inc.) for $1 \mathrm{~h}$ at room temperature. Reactions between antibodies and target proteins were visualized using 3,3'-diaminobenzidine staining. Tissue counterstaining was performed with Mayer's hematoxylin (ready-to-use) for $3 \mathrm{~min}$ at room temperature. The incubation of the tissue sections with $0.01 \mathrm{~mol} / \mathrm{l} \mathrm{PBS}$ instead of primary antibodies was performed as a negative control.

Light microscopy was performed using an Olympus microscope (Olympus Corporation, Tokyo, Japan) and immunohistochemical staining was assessed independently by two experienced clinical pathologists. Nuclear immunostaining for SIRT6 and cytoplasmic immunostaining for NMNAT2 were considered as positive staining. Immunostaining intensity and the percentage of positive cells were evaluated by light microscopy with the following intensity scoring system: 0 (negative), 1 (weak), 2 (moderate) and 3 (strong). The mean percentage of positive cells was quantified from five different visual fields at $\mathrm{x} 200$ magnification using the following percentage positive scoring system: $0,<5 \% ; 1,5-25 \% ; 2,25-50 \% ; 3,50-75 \%$; $4,>75 \%$. The final score for each tissue section was calculated as the immunostaining intensity score ( 0 to 3 ) plus the positive percentage score ( 0 to 4 ) and immunostaining was considered to be positive when the final score was $\geq 3$.

Statistical analysis. The data are presented as the count, percentage or mean \pm standard deviation. Statistical analysis was performed with SPSS version 20.0 (IBM Corp., Armonk, NY, USA). The RT-qPCR and western blotting data were analyzed by paired t-tests. The $\chi^{2}$ or Fisher's exact tests were used for the analysis of immunohistochemistry staining positivity and clinicopathological parameters. The correlation between SIRT6 and NMNAT2 expression was assessed with Spearman's non-parametric correlation. $\mathrm{P}<0.05$ was considered to indicate a statistically significant difference.

\section{Results}

Protein expression of SIRT6 and NMNAT2 in CRC. Fig. 1A includes the results of the western blotting analysis of SIRT6 protein expression in CRC tissue samples, which was lower than the adjacent normal tissues from the same specimen. In contrast, the protein levels of NMNAT2 were increased in CRC when compared with adjacent tissue. The expression of SIRT6 and NMNAT2 protein differed significantly between the CRC and adjacent samples (Fig. 1B and C).

SIRT6 protein expression is localized primarily in nucleus; brown-stained nuclei were observed in the positive sections, as demonstrated in Fig. 2. Positive SIRT6 staining was detected in 25 of 113 cases $(22.12 \%)$ of CRC, compared with 91 of 113 $(80.53 \%)$ adjacent non-cancer specimens; this difference was statistically significant $\left(\chi^{2}=77.152 ; \mathrm{P}<0.01\right)$. 
A

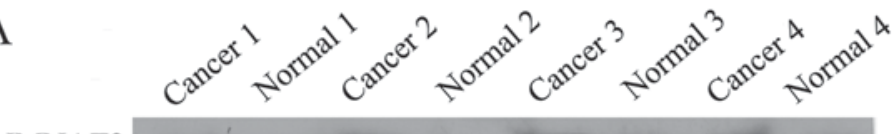

NMNAT2

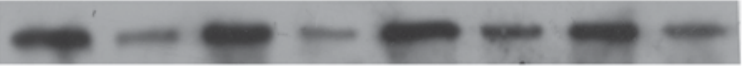

SIRT6

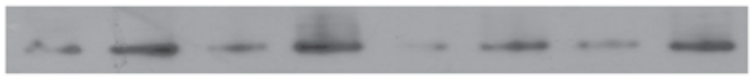

GAPDH
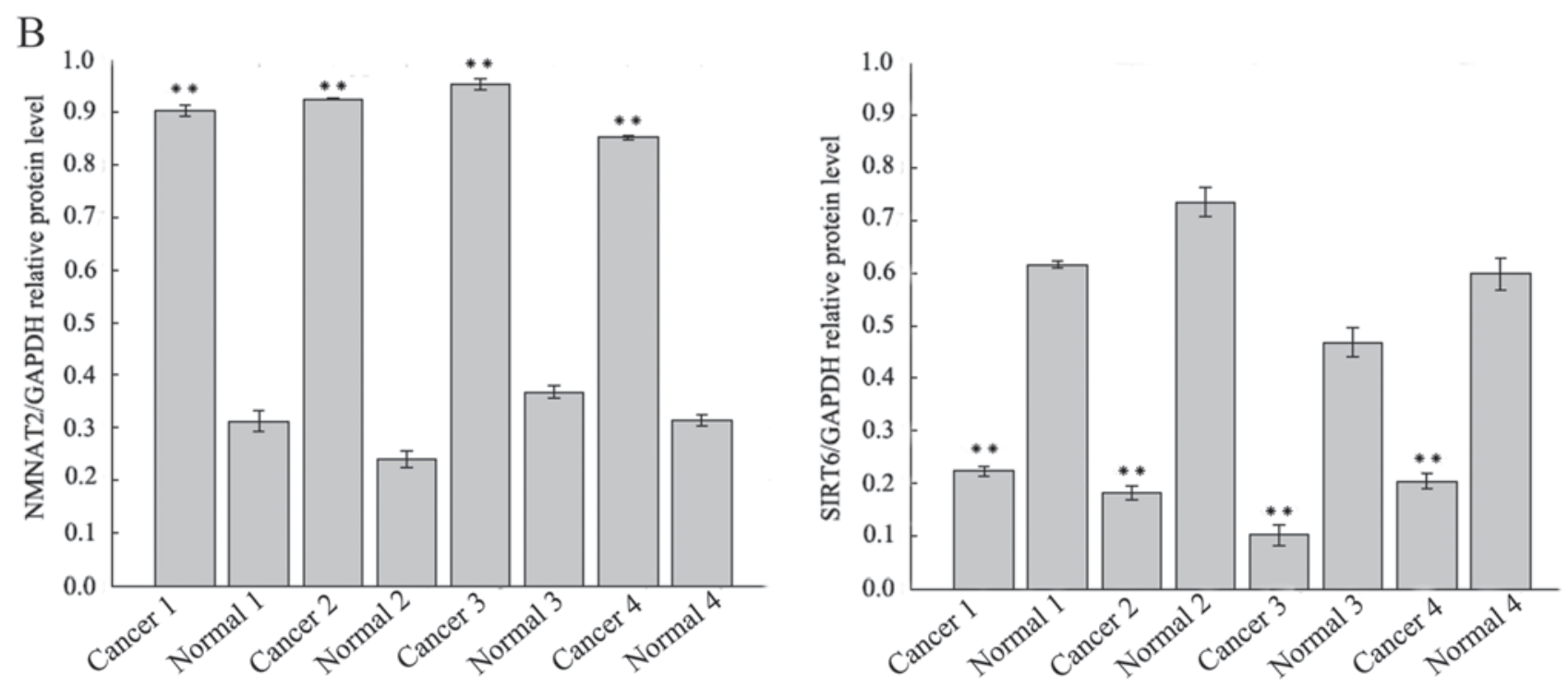

C
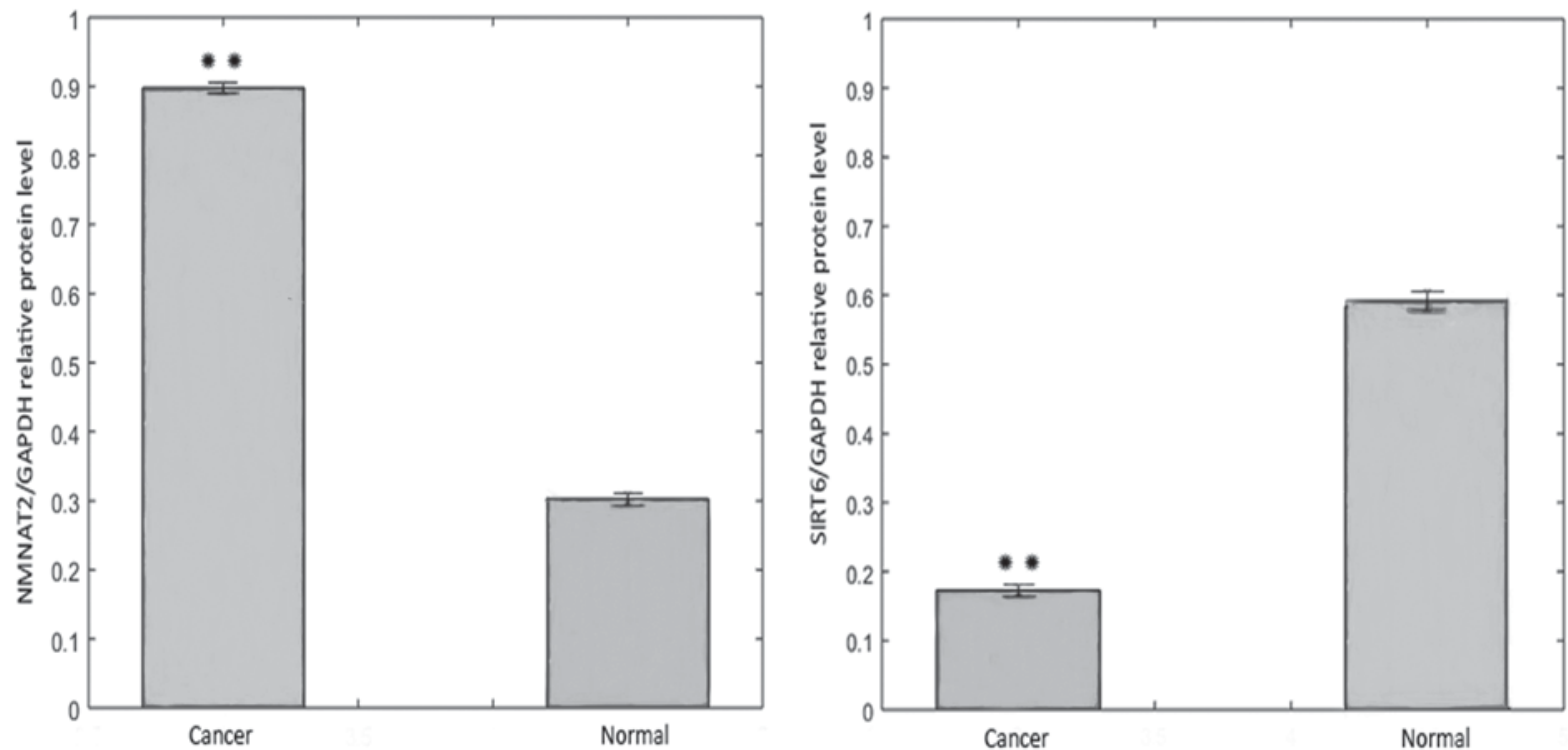

Figure 1. Representative Western blotting results for SIRT6 and NMNAT2, in colorectal cancer tumor and adjacent patient tissues. (A) Protein levels of SIRT6 and NMNAT2 were examined by western blotting. (B) Bar graph of the densitometry values of the relative SIRT6 and NMNAT2 protein expression normalized to GAPDH of 4 representative samples. (C) Bar graph of the densitometry values of the relative SIRT6 and NMNAT2 protein expression normalized to GAPDH of all 29 patients. All data represent the mean \pm standard deviation of three independent experiments. ${ }^{* *}$ P $<0.01$. SIRT6, sirtuin 6; NMNAT2, nicotinamide mononucleotide adenylyltransferase 2 .

The positive cytoplasmic immunohistochemical staining for NMNAT2 protein, which was detected in 82 of 113 cases (72.57\%) of CRC, compared with 36 of $113(31.86 \%)$ adjacent non-cancer specimens is demonstrated in Fig. 3; this difference was also statistically significant $\left(\chi^{2}=37.525\right.$; $\mathrm{P}<0.01)$.
SIRT6 and NMNAT2 mRNA expression levels in CRC. The mRNA expression levels of SIRT6 and NMNAT2 were detected in CRC and adjacent samples using RT-qPCR and were normalized to GAPDH. The expression levels of SIRT6 mRNA in CRC specimens were significantly lower compared with matched adjacent non-cancer tissue specimens $(0.295 \pm 0.161$ vs. 

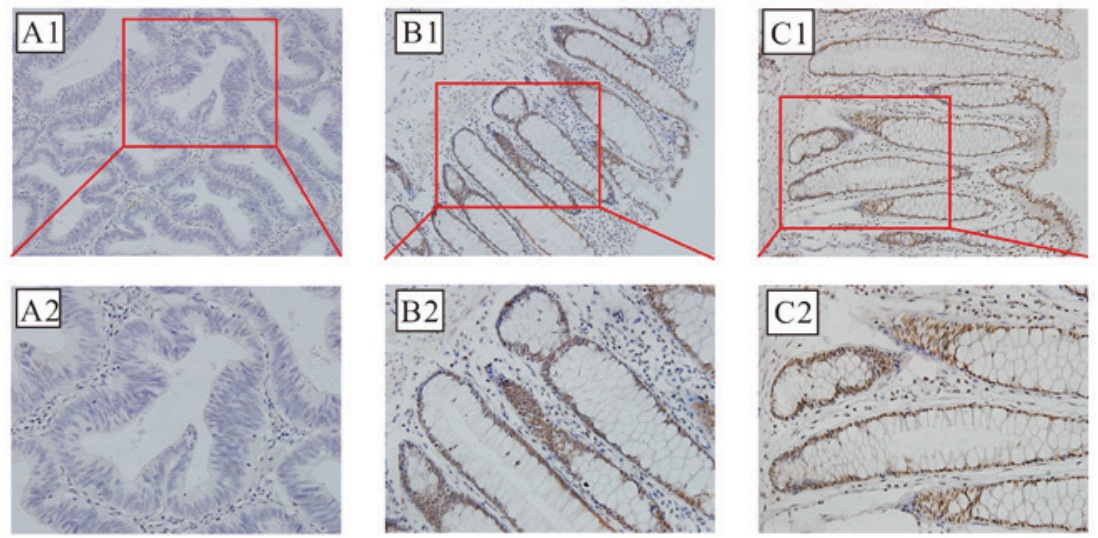

Figure 2. Photomicrographs of the immunohistochemical localization of the expression of SIRT6 in CRC and normal colorectal tissue. Optical photomicrographs of the immunohistochemical staining for SIRT6 in CRC and adjacent colorectal tissues. Positive (brown) immunostaining was identified in the nuclei. Magnification: Upper, x200; lower, x400. (A) Representative image of negative expression. (B) Representative image of positive expression. (C) Representative image of strong positive expression. SIRT6, sirtuin 6; CRC, colorectal cancer.
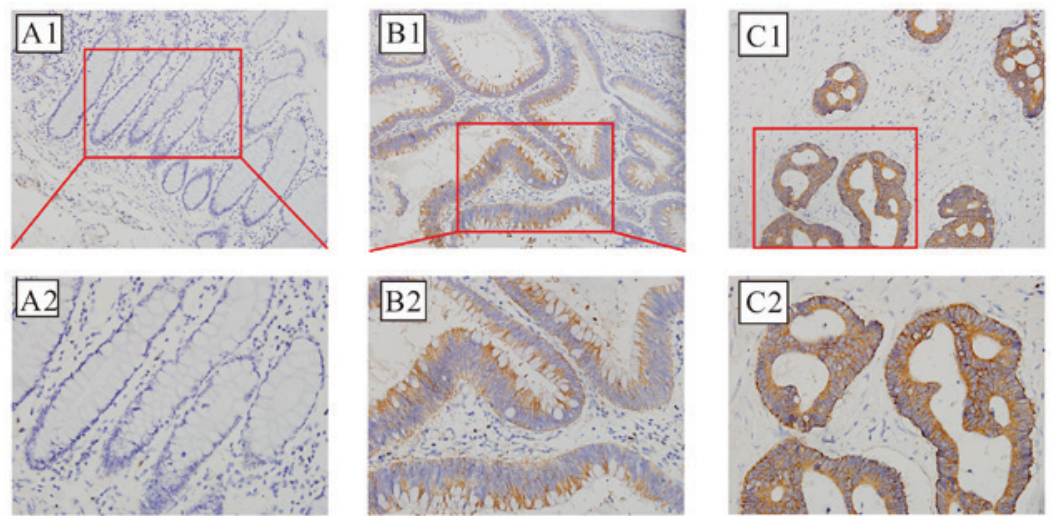

Figure 3. Photomicrographs of the immunohistochemical localization of the expression of NMNAT2 in CRC and normal colorectal tissue. Optical photomicrographs of the immunohistochemical staining for NMNAT2 in CRC and normal colorectal tissues. Positive (brown) immunostaining was identified in the cytoplasm. Magnification: Upper, x200; lower, x400. (A) Representative image of negative expression. (B) Representative image of positive expression. (C) Representative image of strong positive expression. NMNAT2, nicotinamide mononucleotide adenylyltransferase 2; CRC, colorectal cancer.
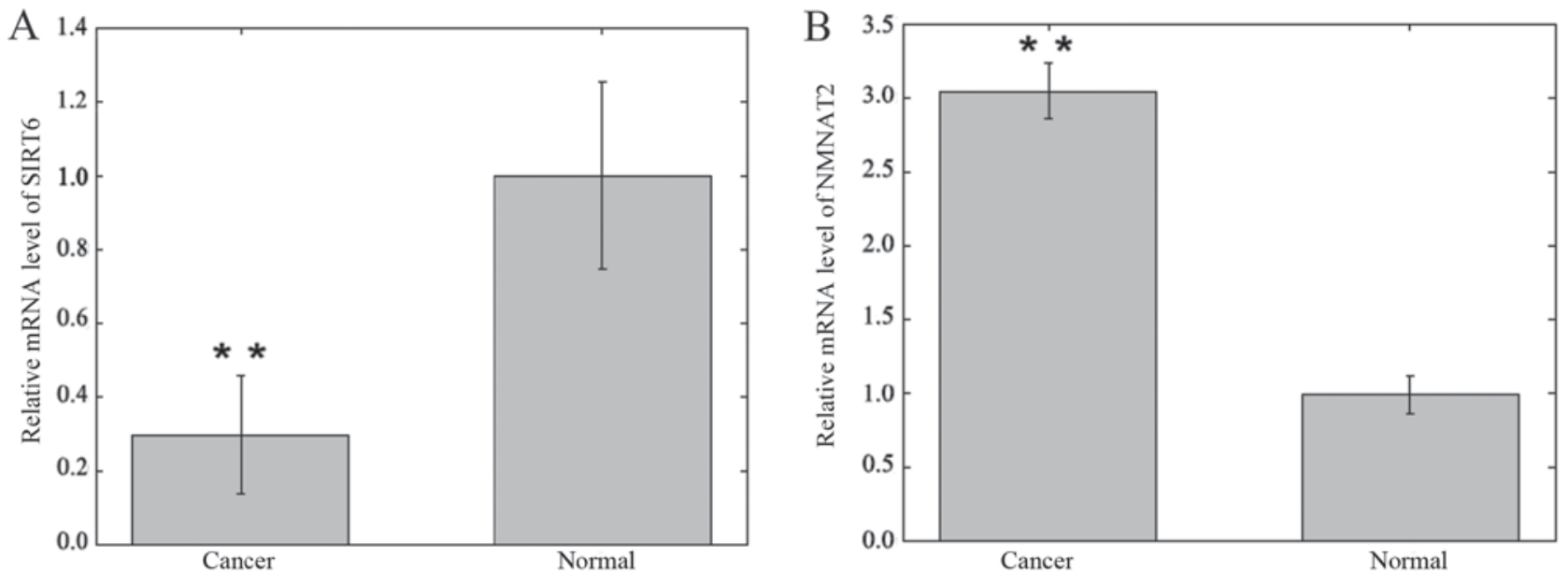

Figure 4. Bar graph of the relative SIRT6 and NMNAT2 mRNA expression in colorectal cancer and adjacent tissue of 29 patients, as analyzed by reverse transcription-quantitative polymerase chain reaction. (A) Relative expression of SIRT6. (B) Relative expression of NMNAT2. All data represent the mean \pm standard deviation of three independent experiments. ${ }^{* *} \mathrm{P}<0.01$. SIRT6, sirtuin 6; NMNAT2, nicotinamide mononucleotide adenylyltransferase 2. 
Table II. Association between the positive expression of SIRT6 and NMNAT2 in colorectal carcinoma tumors as determined with immunohistochemistry with clinical and demographic patient parameters.

\begin{tabular}{|c|c|c|c|c|c|c|c|}
\hline \multirow[b]{2}{*}{ Parameters } & \multirow[b]{2}{*}{ Patients } & \multicolumn{2}{|c|}{ SIRT6, n (\%) } & \multirow[b]{2}{*}{ P-value } & \multicolumn{2}{|c|}{ NMNAT, n (\%) } & \multirow[b]{2}{*}{ P-value } \\
\hline & & Negative & Positive & & Negative & Positive & \\
\hline Total & 113 & 88 & 25 & & 31 & 82 & \\
\hline Sex & & & & 0.527 & & & 0.435 \\
\hline Male & 65 & $52(80.0)$ & $13(20.0)$ & & $16(24.6)$ & $49(75.4)$ & \\
\hline Female & 48 & $36(75.0)$ & $12(25.0)$ & & $15(31.3)$ & $33(68.7)$ & \\
\hline Age, years & & & & 0.529 & & & 0.319 \\
\hline$\leq 64$ & 57 & $43(75.4)$ & $14(24.6)$ & & $18(31.6)$ & $39(68.4)$ & \\
\hline$>64$ & 56 & $45(80.4)$ & 11 (19.6) & & $13(23.2)$ & $43(76.8)$ & \\
\hline Tumor size, $\mathrm{cm}$ & & & & 0.180 & & & 0.332 \\
\hline$\leq 3$ & 50 & $36(72.0)$ & $14(28.0)$ & & $16(32.0)$ & $34(68.0)$ & \\
\hline$>3$ & 63 & $52(82.5)$ & $11(17.5)$ & & $15(23.8)$ & $48(76.2)$ & \\
\hline Differentiation & & & & 0.040 & & & 0.826 \\
\hline Well & 18 & $10(55.6)$ & $8(44.4)$ & & $6(33.3)$ & $12(66.7)$ & \\
\hline Moderate & 87 & $72(82.8)$ & $15(17.2)$ & & $23(26.4)$ & $64(73.6)$ & \\
\hline Poor & 8 & $6(75.0)$ & $2(25.0)$ & & $2(25.0)$ & $6(75.0)$ & \\
\hline Depth of invasion & & & & 0.040 & & & 0.023 \\
\hline $\mathrm{T} 1$ & 7 & 5 (71.4) & $2(28.6)$ & & $4(57.1)$ & $3(42.9)$ & \\
\hline $\mathrm{T} 2$ & 38 & $24(63.2)$ & $14(36.8)$ & & $15(39.5)$ & $23(60.5)$ & \\
\hline $\mathrm{T} 3$ & 17 & $14(82.4)$ & $3(17.6)$ & & $4(23.5)$ & $13(76.5)$ & \\
\hline $\mathrm{T} 4$ & 51 & $45(88.2)$ & $6(11.8)$ & & $8(15.7)$ & $43(84.3)$ & \\
\hline Lymph node metastasis & & & & 0.017 & & & 0.063 \\
\hline N0 & 72 & $51(70.8)$ & $21(29.2)$ & & $24(33.3)$ & $48(66.7)$ & \\
\hline $\mathrm{N} 1+2$ & 41 & $37(90.2)$ & $4(9.8)$ & & $7(17.1)$ & $34(82.9)$ & \\
\hline Distant metastasis & & & & 0.353 & & & 0.775 \\
\hline M0 & 95 & $72(75.8)$ & $23(24.2)$ & & $27(28.4)$ & $68(71.6)$ & \\
\hline M1 & 18 & $16(88.9)$ & $2(11.1)$ & & $4(22.2)$ & $14(77.8)$ & \\
\hline Tumor-node-metastasis stage & & & & 0.032 & & & 0.048 \\
\hline I & 36 & $22(61.1)$ & $14(38.9)$ & & $16(44.4)$ & $20(55.6)$ & \\
\hline II & 30 & $25(83.3)$ & $5(16.7)$ & & $5(16.7)$ & $25(83.3)$ & \\
\hline III & 29 & $25(86.2)$ & $4(13.8)$ & & $6(20.7)$ & $23(79.3)$ & \\
\hline IV & 18 & $16(88.9)$ & $2(11.1)$ & & $4(22.2)$ & $14(77.8)$ & \\
\hline
\end{tabular}

SIRT6, sirtuin 6; NMNAT2, nicotinamide mononucleotide adenylyltransferase 2.

between the expression levels of SIRT6 and NMNAT2. The immunohistochemistry data was further analyzed to confirm the inverse correlation between the protein expression of SIRT6 and NMNAT2 in CRC tissue $(r=-0.246 ; \mathrm{P}<0.01)$. Furthermore, NMNAT2-positive CRC tissue was more likely to be SIRT6-negative than NMNAT2-positive adjacent tissue $(\mathrm{P}<0.05)$, whereas a similar NMNAT2-positive rate was identified between SIRT6-positive CRC and normal samples $(\mathrm{P}>0.05)$. In summary, overexpression of NMNAT2 protein was associated with the reduced expression of SIRT6 protein in CRC tissue.

Association of clinical parameters with SIRT6 and NMNAT2 expression in CRC tissue. Table II summarizes the positive and negative immunohistochemical staining detection of SIRT6 and NMNAT2, and compares the immunopositivity with the demographic and clinicopathological parameters of the 113 patients with CRC from the present study.

The positive expression of SIRT6 protein in CRC tissue was positively associated with the depth of tumor invasion, tumor differentiation grade, lymph node metastasis status and TNM stage $(\mathrm{P}<0.05)$. The positive expression of SIRT6 protein in CRC tissue was more frequent in tumor tissue samples of well-differentiated (low-grade) CRC when compared with moderately differentiated or poorly differentiated (high-grade) CRC. Compared with patients in stage I/II, less positive sections were detected in stage III/IV patients. 
The positive expression of NMNAT2 protein in CRC tissues was positively associated with the depth of tumor invasion and TNM stage $(\mathrm{P}<0.05)$. However, no significant association between SIRT6 or NMNAT2 positive protein expression and the other clinical parameters (including age, sex, tumor size and distant metastasis) were observed in the present study.

\section{Discussion}

The initiation and progression of CRC involves multiple steps, including hyperplasia, metaplasia, pre-invasive carcinoma formation and ultimately, aggressive and invasive cancer. Cells gradually acquire tumor characteristics, including unlimited proliferation, freedom from senescence and apoptosis resistance, leading to uncontrolled growth and tumorigenesis. Normal cells can gain the characteristic abilities of cancer cells following the inactivation of a single tumor suppressor or the activation of a single carcinogenic gene. For insight into the cellular molecular pathogenesis of CRC, specific molecular features associated with colorectal carcinogenesis are in the process of being identified.

The present study included well-characterized human CRC tissues and matched normal colorectal tissues to compare the protein and mRNA expression of SIRT6 and NMNAT2 with western blotting, immunohistochemistry and RT-qPCR. SIRT6 protein and mRNA expression levels were significantly reduced, whereas NMNAT2 protein and mRNA expression levels were significantly increased, in CRC tissue relative to the adjacent tissue $(\mathrm{P}<0.01)$. Immunohistochemistry confirmed a negative correlation between the expression of SIRT6 and NMNAT2 ( $\mathrm{r}=-0.246, \mathrm{P}<0.01)$. The reduced expression of SIRT6 and increased expression of NMNAT2 in CRC tissue were associated with the depth of tumor invasion, TNM stage, tumor differentiation grade and the presence of lymph node metastasis $(\mathrm{P}<0.05)$.

The findings of the present study are supported by previously published in vitro and in vivo studies in animals and human cells. In a mouse model expressing an adenomatosis polyposis coli mutation, SIRT6 deficiency was demonstrated to increase the incidence of invasive colonic adenocarcinoma (17). The knockout of the SIRT6 gene from mouse embryonic fibroblasts enhanced the proliferative capability of cells and induced tumor formation without activating oncogenes in a severe combined immune deficiency mouse model (16). In human endometrial and hepatocellular cancer cell lines, the increased expression of SIRT6 suppressed proliferation and induced apoptosis by inhibiting the expression of the anti-apoptotic protein, surviving $(28,29)$. In the present study, it was demonstrated that in human CRC, increasing tumor grade and stage was associated with the decreased or deficient expression of SIRT6. This result may support the hypothesis that SIRT6 acts as a tumor-suppressor gene in human colorectal tissue. In support of this view, SIRT6 was previously demonstrated to affect cancer cell proliferation by suppressing the transcriptional activity of c-Myc, a known oncogene (30).

However, other studies have indicated that there may be other metabolic mechanisms involved in the tumor-suppressive effects of SIRT6 $(31,32)$. The 'Warburg effect' is the reprogramming of the cellular energy metabolism to support continuous cell growth and proliferation in malignancy $(33,34)$. It was previously demonstrated that the deletion of SIRT6 may activate an energy metabolism program that promotes tumorigenesis independently of any other transforming events (17). The roles of SIRT6 as a metabolic enzyme and a potential regulator of cancer cell metabolism remain to be investigated.

NMNAT2 is important in cell metabolism, and so the increased expression of NMNAT2 in rapidly dividing cancer cells would be expected $(21,22)$. SIRT3 has been demonstrated to be necessary for the deacetylation of NMNAT2 in lung cancer cells, which promotes cell proliferation and growth through affecting energy metabolism, so it is logical that SIRT6 may have a similar role in CRC (35). However, the present study has also demonstrated that the mechanism underlying SIRT6 as a tumor suppressor in CRC may also involve regulating the transcription or expression of NMNAT2. The protein and the mRNA levels of NMNAT2 were upregulated in CRC tissue when compared with the normal colorectal tissue samples, and a negative correlation between SIRT6 and NMNAT2 was identified. This may indicate that NMNAT2 is involved in the tumorigenesis and development of CRC by maintaining intracellular NAD, fuelling rapid cell growth and proliferation. Furthermore, the results indicate that the expression of NMNAT2 may be regulated, at least partly, by SIRT6 through mRNA and protein regulatory mechanisms in CRC.

In the present study, the expression levels of SIRT6 and NMNAT2 in CRC were studied in relation to patient demographics and clinicopathological parameters using immunohistochemistry, a technique that is part of routine histopathological diagnosis and prognostic evaluation. The histological protein expression of SIRT6 and NMNAT2 in human CRC was negatively correlated with the presence of $\mathrm{CRC}$ and with $\mathrm{CRC}$ grade $(\mathrm{r}=-0.246 ; \mathrm{P}<0.01)$. These immunohistochemistry findings may support the hypothesis that the increased expression of NMNAT2 and reduced expression of SIRT6 were associated with CRC progression, and that the downregulation of SIRT6 may promote the expression of NMNAT2. Further studies are recommended to investigate the potential association between SIRT6 and NMNAT2 in CRC, and other malignant tumor types, to provide new insights into the inhibition of tumor progression by affecting the tumor cell metabolism.

The present study has potential clinical implications for $\mathrm{CRC}$ and other forms of malignant disease, indicating that further studies should be undertaken. In patients with ovarian cancer or hepatocellular carcinoma, the decreased expression of SIRT6 was previously associated with accelerated tumor cell growth and poor clinical prognosis $(14,36)$. Previous studies have demonstrated an oncogenic role for activated metabolic enzyme adaptations during cancer progression, including the serine biosynthetic rate-limiting enzyme D-3-phosphoglycerate dehydrogenase, and overexpression of the glycine-metabolizing enzyme glycine decarboxylase (37-39). There is evidence that the inhibition of aberrant glycolysis may be a potential treatment strategy for cancer, with the enzymes associated with the dysfunction of NAD metabolism being suggested as treatment targets for obesity, diabetes and cancer (40). Poly(ADP) ribose polymerase inhibitors have also been investigated 
for chemoprevention, radiosensitization and as anticancer agents (41). This supports the potential for NMNAT2 as a diagnostic and therapeutic target in CRC.

The present study had several limitations. It should be acknowledged that this was a preliminary study that investigated a small number of tumor samples from a limited number of colorectal cancer surgical units. Although tissue sampling was performed with the assistance of experienced pathologists, it was not possible to exclude the possibility that the 'normal' tissues were inflamed, adenomatous or dysplastic, or contained micrometastases, as sampling was performed visually. The tissues sampled were collected and stored according to a standard protocol to ensure that the integrity of tissue proteins and mRNA were preserved. However, in a busy surgical and surgical pathology unit, delays can occur between the surgical removal of a specimen and tissue sampling that cannot easily be controlled. Furthermore, the immunohistochemical staining of the tumor and normal cells was evaluated by skilled pathologists, but the scoring was performed 'by eye', which may be subject to grading bias; this limitation could be overcome in future studies with the use of image analysis and objective immunohistochemical quantification techniques.

In conclusion, the findings of this preliminary study demonstrated that the increased expression of NMNAT2 and reduced expression of SIRT6 were associated with the progression of CRC. It is possible that the downregulation of SIRT6 may promote the expression of NMNAT2. Further studies are required to characterize the role of NMNAT2 and SIRT6 as potential diagnostic and prognostic biomarkers and as targets for therapy in CRC, and other types of malignant tumor.

\section{Acknowledgements}

Not applicable.

\section{Funding}

The present study was supported by the Natural Science Foundation of Guangdong Province, China (grant no., 2014 a030313295).

\section{Availability of data and materials}

All data generated or analyzed during the present study are included in this published article.

\section{Authors' contributions}

JQ was responsible for designing the present study, for conducting the main experiments and compiling the manuscript. QD was involved in preparing the sample sections and for protein extraction. LW collated and analyzed the data. RC, DZ and LX contributed to the sample collection. CC and JY were involved in designing the study and revising the manuscript critically for important intellectual content.

\section{Ethics approval and consent to participate}

The present study, including the protocols for collection of tissue specimens and analysis of clinical information, was approved by the Institutional Review Board of Nanfang Hospital and Zhujiang Hospital of the Southern Medical University (Guangzhou, China).

\section{Patient consent for publication}

All patients included in this study were informed of the study protocol and requirements, and provided signed informed consent to participate in the study.

\section{Competing interests}

The authors declare that they have no competing interests.

\section{References}

1. Siegel RL, Miller KD and Jemal A: Cancer statistics, 2016. CA Cancer J Clin 66: 7-30, 2016.

2. Siegel R, Desantis C and Jemal A: Colorectal cancer statistics, 2014. CA Cancer J Clin 64: 104-117, 2014.

3. Favoriti P, Carbone G, Greco M, Pirozzi F, Pirozzi RE and Corcione F: Worldwide burden of colorectal cancer: A review. Updates Surg 68: 7-11, 2016.

4. Torre LA, Bray F, Siegel RL, Ferlay J, Lortet-Tieulent J and Jemal A: Global cancer statistics, 2012. CA Cancer J Clin 65: 87-108, 2015.

5. Rabeneck L, Horton S, Zauber AG and Earle C: Colorectal Cancer. In: Cancer: Disease Control Priorities, Third Edition (Volume 3). Gelband H, Jha P, Sankaranarayanan R and Horton S (eds). The International Bank for Reconstruction and Development/The World Bank (C) 2015 International Bank for Reconstruction and Development/The World Bank, Washington (DC), 2015.

6. Zhu AX, Kudo M, Assenat E, Cattan S, Kang YK, Lim HY, Poon RT, Blanc JF, Vogel A, Chen CL, et al: Effect of everolimus on survival in advanced hepatocellular carcinoma after failure of sorafenib: The EVOLVE-1 randomized clinical trial. JAMA 312: 57-67, 2014.

7. Michan S and Sinclair D: Sirtuins in mammals: Insights into their biological function. Biochem J 404: 1-13, 2007.

8. Bruzzone S, Parenti MD, Grozio A, Ballestrero A, Bauer I, Del Rio A and Nencioni A: Rejuvenating sirtuins: The rise of a new family of cancer drug targets. Curr Pharm Des 19: 614-623, 2013.

9. Lerrer B, Gertler AA and Cohen HY: The complex role of SIRT6 in carcinogenesis. Carcinogenesis 37: 108-118, 2016.

10. Michishita E, McCord RA, Berber E, Kioi M, Padilla-Nash H, Damian $M$, Cheung $\mathrm{P}$, Kusumoto $\mathrm{R}$, Kawahara TL, Barrett JC, et al: SIRT6 is a histone H3 lysine 9 deacetylase that modulates telomeric chromatin. Nature 452: 492-496, 2008.

11. Tennen RI and Chua KF: Chromatin regulation and genome maintenance by mammalian SIRT6. Trends Biochem Sci 36: 39-46, 2011.

12. Toiber D, Erdel F, Bouazoune K, Silberman DM, Zhong L, Mulligan P, Sebastian C, Cosentino C, Martinez-Pastor B, Giacosa S, et al: SIRT6 recruits SNF2H to DNA break sites, preventing genomic instability through chromatin remodeling. Mol Cell 51: 454-468, 2013.

13. Cea M, Cagnetta A, Adamia S, Acharya C, Tai YT, Fulciniti M, Ohguchi H, Munshi A, Acharya P, Bhasin MK, et al: Evidence for a role of the histone deacetylase SIRT6 in DNA damage response of multiple myeloma cells. Blood 127: 1138-1150, 2016.

14. Zhang G, Liu Z, Qin S and Li K: Decreased expression of SIRT6 promotes tumor cell growth correlates closely with poor prognosis of ovarian cancer. Eur J Gynaecol Oncol 36: 629-632, 2015.

15. Yu SS, Cai Y, Ye JT, Pi RB, Chen SR, Liu PQ, Shen XY and Ji Y: Sirtuin 6 protects cardiomyocytes from hypertrophy in vitro via inhibition of NF- $\mathrm{B}$-dependent transcriptional activity. Br J Pharmacol 168: 117-128, 2013.

16. Sundaresan NR, Vasudevan P, Zhong L, Kim G, Samant S, Parekh V, Pillai VB, Ravindra PV, Gupta M, Jeevanandam V, et al: The sirtuin SIRT6 blocks IGF-Akt signaling and development of cardiac hypertrophy by targeting c-Jun. Nat Med 18: 1643-1650, 2012. 
17. Sebastian C, Zwaans BM, Silberman DM, Gymrek M, Goren A, Zhong L, Ram O, Truelove J, Guimaraes AR, Toiber D, et al: The histone deacetylase SIRT6 is a tumor suppressor that controls cancer metabolism. Cell 151: 1185-1199, 2012.

18. Barretina J, Caponigro G, Stransky N, Venkatesan K, Margolin AA, Kim S, Wilson CJ, Lehár J, Kryukov GV, Sonkin D, et al: The Cancer Cell Line Encyclopedia enables predictive modelling of anticancer drug sensitivity. Nature 483: 603-607, 2012.

19. Mayer PR, Huang N, Dewey CM, Dries DR, Zhang H and Yu G: Expression, localization, and biochemical characterization of nicotinamide mononucleotide adenylyltransferase 2 . J Biol Chem 285: 40387-40396, 2010.

20. Jayaram HN, Kusumanchi P and Yalowitz JA: NMNAT expression and its relation to NAD metabolism. Curr Med Chem 18 1962-1972, 2011.

21. Mouchiroud L, Houtkooper RH and Auwerx J: NAD ${ }^{+}$metabolism: A therapeutic target for age-related metabolic disease. Crit Rev Biochem Mol Biol 48: 397-408, 2013.

22. Cairns RA, Harris IS and Mak TW: Regulation of cancer cell metabolism. Nat Rev Cancer 11: 85-95, 2011

23. Zhong L, D'Urso A, Toiber D, Sebastian C, Henry RE, Vadysirisack DD, Guimaraes A, Marinelli B, Wikstrom JD, Nir T, et al: The histone deacetylase Sirt6 regulates glucose homeostasis via Hif1alpha. Cell 140: 280-293, 2010.

24. Bauer I, Grozio A, Lasigliè D, Basile G, Sturla L, Magnone M, Sociali G, Soncini D, Caffa I, Poggi A, et al: The NAD+-dependent histone deacetylase SIRT6 promotes cytokine production and migration in pancreatic cancer cells by regulating $\mathrm{Ca} 2+$ responses. J Biol Chem 287: 40924-40937, 2012

25. Resch A and Langner C: Lymph node staging in colorectal cancer: Old controversies and recent advances. World J Gastroenterol 19 8515-8526, 2013

26. Van Cutsem E and Ducreux M: Colorectal and gastric cancer in 2015: The development of new agents and molecular classifications. Nat Rev Clin Oncol 13: 69-70, 2016.

27. Livak KJ and Schmittgen TD: Analysis of relative gene expression data using real-time quantitative PCR and the 2(-Delta Delta C(T)) method. Methods 25: 402-408, 2001.

28. Fukuda T, Wada-Hiraike O, Oda K, Tanikawa M, Makii C, Inaba K, Miyasaka A, Miyamoto Y, Yano T, Maeda D, et al: Putative tumor suppression function of SIRT6 in endometrial cancer. FEBS Lett 589: 2274-2281, 2015.

29. Zhang ZG and Qin CY: Sirt6 suppresses hepatocellular carcinoma cell growth via inhibiting the extracellular signal-regulated kinase signaling pathway. Mol Med Rep 9: 882-888, 2014.

30. Lin Z, Yang H, Tan C, Li J, Liu Z, Quan Q, Kong S, Ye J, Gao B and Fang D: USP10 antagonizes c-Myc transcriptional activation through SIRT6 stabilization to suppress tumor formation. Cell Rep 5: 1639-1649, 2013.
31. Kleszcz R, Paluszczak J and Baer-Dubowska W: Targeting aberrant cancer metabolism-The role of sirtuins. Pharmacol Rep 67: 1068-1080, 2015

32. Xiong X, Wang G, Tao R, Wu P, Kono T, Li K, Ding WX, Tong X, Tersey SA, Harris RA, et al: Sirtuin 6 regulates glucose-stimulated insulin secretion in mouse pancreatic beta cells. Diabetologia 59: 151-160, 2016.

33. Vander Heiden MG, Locasale JW, Swanson KD, Sharfi H, Heffron GJ, Amador-Noguez D, Christofk HR, Wagner G, Rabinowitz JD, Asara JM and Cantley LC: Evidence for an alternative glycolytic pathway in rapidly proliferating cells. Science 329: 1492-1499, 2010.

34. Hsu PP and Sabatini DM: Cancer cell metabolism: Warburg and beyond. Cell 134: 703-707, 2008

35. Li H, Feng Z, Wu W, Li J, Zhang J and Xia T: SIRT3 regulates cell proliferation and apoptosis related to energy metabolism in non-small cell lung cancer cells through deacetylation of NMNAT2. Int J Oncol 43: 1420-1430, 2013.

36. Marquardt JU, Fischer K, Baus K, Kashyap A, Ma S, Krupp M, Linke M, Teufel A, Zechner U, Strand D, et al: Sirtuin-6-dependent genetic and epigenetic alterations are associated with poor clinical outcome in hepatocellular carcinoma patients. Hepatology 58: 1054-1064, 2013.

37. Locasale JW, Grassian AR, Melman T, Lyssiotis CA, Mattaini KR, Bass AJ, Heffron G, Metallo CM, Muranen T, Sharfi $\mathrm{H}$, et al: Phosphoglycerate dehydrogenase diverts glycolytic flux and contributes to oncogenesis. Nat Genet 43: 869-874, 2011.

38. Possemato R, Marks KM, Shaul YD, Pacold ME, Kim D, Birsoy K, Sethumadhavan S, Woo HK, Jang HG, Jha AK, et al: Functional genomics reveal that the serine synthesis pathway is essential in breast cancer. Nature 476: 346-350, 2011.

39. Zhang WC, Shyh-Chang N, Yang H, Rai A, Umashankar S, Ma S, Soh BS, Sun LL, Tai BC, Nga ME, et al: Glycine decarboxylase activity drives non-small cell lung cancer tumor-initiating cells and tumorigenesis. Cell 148: 259-272, 2012.

40. Cantó C, Houtkooper RH, Pirinen E, Youn DY, Oosterveer MH, Cen Y, Fernandez-Marcos PJ, Yamamoto H, Andreux PA, Cettour-Rose $\mathrm{P}$, et al: The $\mathrm{NAD}(+)$ precursor nicotinamide riboside enhances oxidative metabolism and protects against high-fat diet-induced obesity. Cell Metab 15: 838-847, 2012.

41. Mégnin-Chanet F, Bollet MA and Hall J: Targeting poly(ADP-ribose) polymerase activity for cancer therapy. Cell Mol Life Sci 67: 3649-3662, 2010.

This work is licensed under a Creative Commons Attribution-NonCommercial-NoDerivatives 4.0 International (CC BY-NC-ND 4.0) License. 